\title{
LITERATURE SURVEY FOR 3D RECONSTRUCTION OF BRAIN MRI
} IMAGES

\author{
Megha Borse ${ }^{1}$, S.B. Patil ${ }^{2}$, B.S.Patil ${ }^{3}$ \\ ${ }^{1}$ Assistant Professor, Dept. of Electronics \&Telecommunication, Cummins College of Engineering for Women, \\ Maharashtra,India,bmeghas@gmail.com \\ ${ }^{2}$ Professor, Department of Electronics, Dr. J.J. Magdum College of Engineering, Maharashtra, India, \\ sbpjjm_2004@yahoo.co.in \\ ${ }^{3}$ Associate Professor, Head Department of Information and Technology, PVPIT College of Engineering, \\ Maharashtra, India, bsp_pvp2004@yahoo.co.in
}

\begin{abstract}
Since Doctors had only the 2D Image Data to visualize the tumors in the MRI images, which never gave the actual feel of how the tumor would exactly look like. The doctors were deprived from the exact visualization of the tumor the amount of the tumor to be removed by operation was not known, which caused a lot of deformation in the faces and structure of the patients face or skull. The diversity and complexity of tumor cells makes it very challenging to visualize tumor present in magnetic resonance image (MRI) data. Hence to visualize the tumor properly $2 D$ MRI image has to be converted to $3 D$ image. With the development of computer image processing technology, three-dimensional (3D) visualization has become an important method of the medical diagnose, it offers abundant and accurate information for medical experts. Three-dimensional (3-D) reconstruction of medical images is widely applied to tumor localization; surgical planning and brain electromagnetic field computation etc. The brain MR images have unique characteristics, i.e., very complicated changes of the gray-scales and highly irregular boundaries. Traditional 3-D reconstruction algorithms are challenged in solving this problem. Many reconstruction algorithms, such as marching cubes and dividing cubes, need to establish the topological relationship between the slices of images. The results of these traditional approaches vary depending on the number of input sections, their positions, the shape of the original body and the applied interpolation technique. These make the task tedious and time-consuming. Moreover, satisfied reconstruction result may not even be obtained when the highly irregular objects such as the encephalic tissues are considered. Due to complexity and irregularity of each encephalic tissue boundary, threedimensional (3D) reconstruction for MRI image is necessary. A Literature survey is done to study different methods of $3 D$ reconstruction of brain images from MRI images.
\end{abstract}

Keywords: 3-D reconstruction, region growing, segmentation method, immune algorithm (IA), one class support vector machine (OCSVM) and sphere shaped support vector machine (SSSVM). $* * *$

\section{INTRODUCTION}

Human brain is the most vital and complex organ of our central nervous system. It is composed of 7 different types of tissues. It is really important to convert available 2-D MRI images to a 3-D brain image to determine if there are any irregularities. 3-D reconstruction of medical images is widely applied to tumor localization, surgical planning and removing the need for rescanning. The brain MRI images consist of wide range of the gray-scales and highly irregular boundaries. So it is difficult to classify different tissues using existing methods. These methods are tedious and time-consuming and most of the times don't give satisfactory results.

Previously, the realization of 3D visualization was mainly by two reconstruction methods, one is surface rendering and the other is volume rendering.

\section{SURFACE RENDERING [6]}

In surface rendering method [6] suggested by Jayram Udupa, Hsiu-Mei Hung, Keh-Shih Chuang a threshold value of grayscale density is chosen by the operator. Then a threshold level is set, using edge detection image processing algorithms. From this, a 3-dimensional model can be constructed and displayed on screen. Multiple models can be constructed from various different thresholds, allowing different colors to represent each anatomical component such as bone, muscle, and cartilage. However, the interior structure of each element is not visible in this mode of operation. Surface rendering has limitation, such that it will only display surfaces which meet a threshold density and will only display the surface that is closest to the imaginary viewer. 


\section{VOLUME RENDERING [3]}

In volume rendering method [3] by Cheng-Sheng Hung, Chien-Feng Huang, Ming Ouhyoung, transparency and colors are used to allow a better representation of the volume to be shown in a single image - e.g. the bones of the pelvis could be displayed as semi-transparent, so that even at an oblique angle, one part of the image does not conceal another. Where different structures have similar threshold density, it can become impossible to separate them simply by adjusting volume rendering parameters.

Literature survey is done to find out the more effective method of $3 \mathrm{D}$ reconstruction of brain MRI images.

\section{OTSUS THRESHOLD TECHNIQUE [1]}

Tthe next method studied [1] is, "3-D Reconstruction of Tumors in MRI Images" by K Narayanan1, Yogesh Karunakar 2.This method involves implementation of MRI brain images by OTSUs threshold technique and various morphological operations and Patch functions in MATLAB for reconstructing 3D image from a set of 2D tumor images. Extensive use of custom made user interface that provides for ease of user interaction and visualization of reconstructed data. The volume of the tumor is also estimated based on the computation of these images. Doctors and Radiologists can process thousands of image samples using this method and save on time per day. User friendly software for processing the MRI image was developed. This software applied to real data has given fair results in terms of volume reconstruction of tumors. However, in some cases when tumor contrast was relatively poor, or the image pixel values of the tumor region were very different from their expected values, software could not reconstruct the volume with good reproducibility. This further shows the importance of acquiring the high quality MRI scans with sufficiently good resolution and contrast for automated volume measurement and display using the present software of volume reconstruction of tumors. However, in some cases, when tumor contrast was relatively poor, or the image pixel values of the tumor region were very different from their expected values, software could not reconstruct the volume with good reproducibility. Fig. 1 gives $3 \mathrm{D}$ view of tumor using this method

\section{FUZZY CLASSIFICATION METHOD [2]}

The next [2] method suggested by Hassan Khotanloua, Olivier Colliotb, Jamal Atifc, Isabelle Blocha, is mentioned in the paper titled "3D brain tumor segmentation in MRI using fuzzy classification." The automated brain tumor segmentation method developed is composed of two phases: initialization and refinement. In the first phase, tumor is detected and segmented. To perform this operation, the brain is segmented by a combination of histogram analysis, morphological operations and symmetry analysis. Within the brain, the tumor is then detected using a fuzzy classification method or symmetry analysis and some morphological operations. The first method relies on the assumption that the tumor appears in the image with specific gray levels, corresponding to an additional class. The second method relies on the assumption that the brain is roughly symmetrical in shape, and that tumors can be detected as areas that deviate from the symmetry assumption when looking at gray levels. This detection provides the initialization for a more precise segmentation step, performed in the second stage, using a parametric deformable model constrained by fuzzy spatial relations. This allows representing explicitly relations between the tumors and surrounding tissues, thus reinforcing the robustness of the method. All processing steps are performed in 3D.

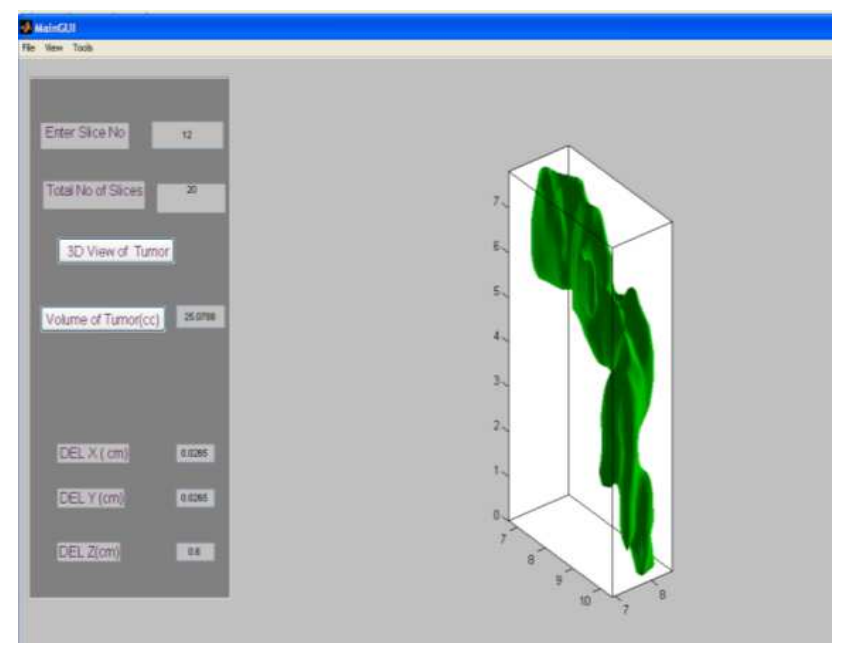

Fig 1 3D View of Tumor

Several sources of imprecision are taken into account in the used method. Imprecision is inherently present in the images, due to the observed phenomenon itself (imprecise limits of pathological areas for instance), to the acquisition system and the numerical reconstruction process (leading to spatial and intensity imprecision). Moreover, available knowledge is also prone to imprecision. For instance the constant order of the gray levels of the main brain tissues are exploited, but the exact range of values of each tissue is imprecise. Here use of spatial relations, expressed in linguistic form, such as "near the tumor", which cannot be modelled in a precise way. All these reasons justify the use of fuzzy models in several steps of the proposed approach (fuzzy classification based on gray levels, models of spatial relations).

In this paper a hybrid segmentation method is developed that uses both region and boundary information of the image to segment the tumor. A fuzzy classification method and a symmetry analysis method are compared to detect the tumors and a deformable model constrained by spatial relations for segmentation refinement is used. This work shows that the 
symmetry plane is a useful feature for tumor detection. In comparison with other methods, approach of this method has some advantages such as automation (in the symmetry analysis method, a reduced interaction is required to select the appropriate peaks in the difference histogram), and more generality with respect to the wide range of tumors. A limitation of this approach is that the symmetry analysis may fail in the case of a symmetrical tumor across the mid-sagital plane. However, this case is very rare. Fig.2 shows comparison of FPCM method with manual and symmetry analysis.

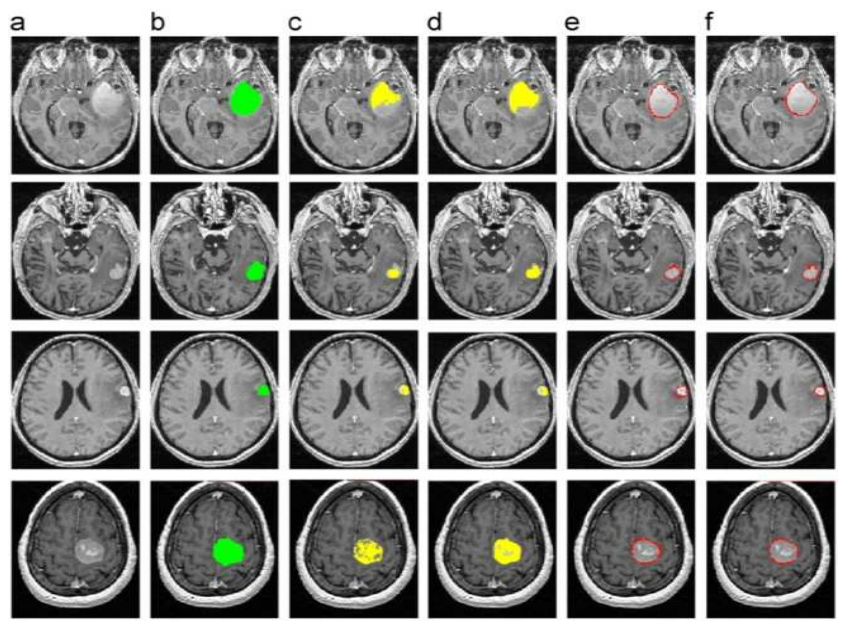

Fig2. Comparison of FPCM method with manual and symmetry analysis segmentation results (a) One axial slice of the original image. (b) Manual segmentation. (c) Initial detection by FPCM. (d) Initial detection by symmetry analysis. (e) Final result by FPCM method and deformable model. (f) Final result by symmetry analysis and spatial relation constrained deformable model.

\section{USING MATLAB [4]}

The next method studied by Duška Kleut, Milorad Jovanović and prof. Dr Branimir Reljin is, "3D Visualisation of MRI images using MATLAB". The goal of this work was to show the possibility of such reconstruction using a wide range programme such as MATLAB. Solution is displayed through an example which does not allow virtual "entering" into the 3D structure, but enables slicing out desired volume to a size of one pixel of the original images. Tomographic layers at the appropriate depth will be displayed on both sides of chosen parallelopiped. Sets of images originating from two modalities were placed at disposal.

Images used in this project originate from the device, having greater resolution. . Two sets were used, in both sagital and transversal plain, with the emphasis on the latter one. The example displayed in this paper shows how a powerful tool like MATLAB can be used for 3D visualisation of 3D tomographic images. It is mentioned that with further work on this realisation it could be possible to create the effect of entering the srtucture in such a way to observe inner volumes. MATLAB supports this function, but working with matrixes this large would be very time consuming for home computers. Fig.3 gives 3D image of reconstructed head using this method.

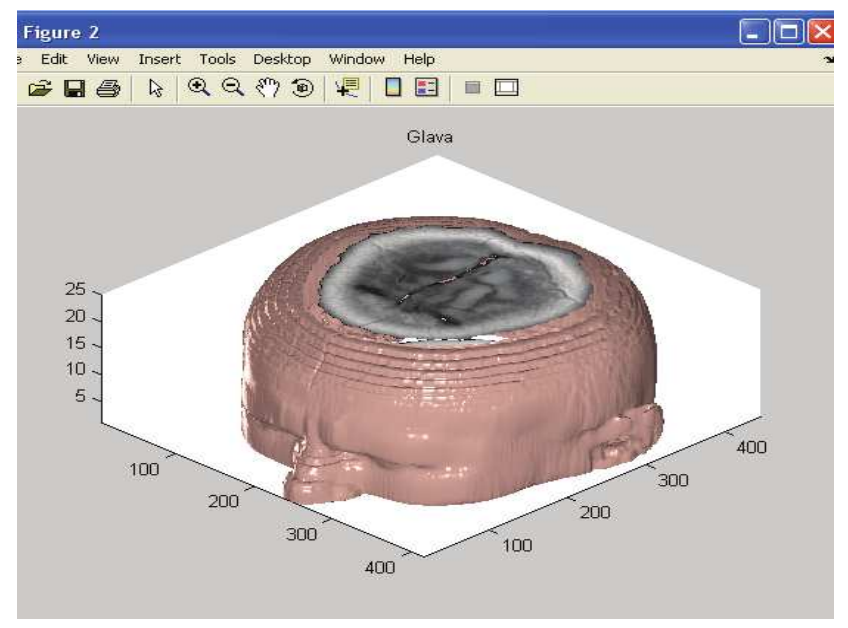

Fig 3: Reconstructed head

\section{USE OF ONE CLASS SVM [5]}

In the next method by Lei Wang, Guizhi Xu, Lei Guo, Xuena Liu and Shuo Yang, "3D Reconstruction of Head MRI Based on One Class Support Vector Machine with Immune algorithm", SVM algorithm based on the statistical learning theory is used. It was initially developed to solve the problem of classification; subsequently they have been extended to regression problems. The principle of this theory is mapping the input data into a higher dimensional feature space by a suitable choice of kernel function. The core idea of SVM is to deal with the problem of separating the set of samples into two respective classes by a hyper plane. The desired situation is they are optimally separated without error and the distance between the closest vector and the hyper plane is maximal.

In head MRI, the boundary of each encephalic tissue is very complicated and irregular, moreover, each tissue has a huge number of samples, and the numbers of the samples are unbalanced. To solve the problems mentioned above, in this paper, one class SVM (OCSVM) was introduced for reconstructing the $3 \mathrm{D}$ models of seven kinds of encephalic tissues. The OCSVM fits a tight hyper sphere in the nonlinearly transformed feature space including most of the target samples which were used to reconstruct the 3D models. Then, 3D reconstruction was fulfilled. To practice the training, SVM was utilized. Because of the model section is hard to be decided, immune algorithm is presented to solve the optimization problem. Aiming at choosing the optimal parameters from large collections of information, 10-fold 
cross validation was introduced. This article is organized by these ideas. The results demonstrate that the reconstruction of head MRI using OCSVM with these ideas has a perfect performance, either in the efficiency or accuracy. Fig.4 shows the reconstruction results of CSF and OCS using one class support vector machine

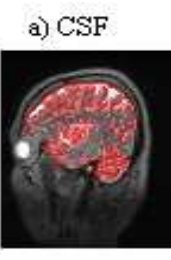

b)Entity
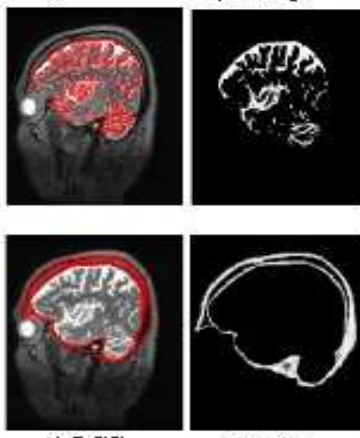

e) $\mathrm{OCS}$

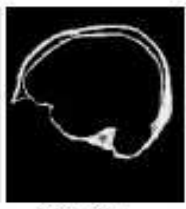

f)Entity
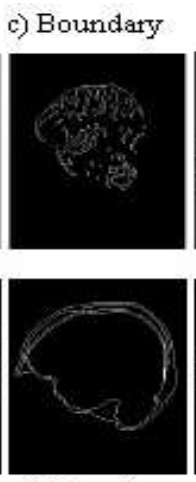

g)Boundary d) $3 \mathrm{D}$
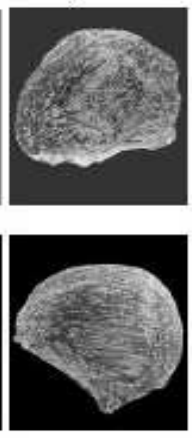

h) $3 \mathrm{D}$
Fig 4 Reconstruction results of CSF and OCS by OCSVM

\section{SEGMENTED STRUCTURES [7]}

The next [7] method suggested by Paul Herghelegiu, Marius Gavrilescu, and Vasile Manta is, "Visualization of Segmented Structures in 3D Multimodal Medical Data Sets."The simultaneous inspection of images obtained using different medical scanning methods represents a common practice for accurate medical diagnosis. The term multimodality refers to multiple medical data sets obtained by scanning a patient with the same method at different time moments or with different scanning techniques." Recent research efforts in computer graphics have attempted to solve the problem of visualizing multimodal data in the same scene, for a better understanding of human anatomy or for pathology tracking. This paper proposes a method of integrating segmented structures from a contrast enhanced MRI sequence into the volume reconstructed from the slices of another MRI sequence obtained with different scanning parameters. A direct volume rendering (DVR) approach is used to represent focus and context information from the 3D data. The presented approach aims to help physicians in understanding pathologies and in the process of accurate diagnosis establishment. In this paper, a method for visualizing a previously segmented structure from T1 contrast enhanced MRI sequence into the reconstructed volume from the slices obtained from T2 weighted MRI sequence is developed. A DVR approach was used to represent focus and context information from within the data. Although the input data has a significant degree of complexity, the visualization methods run on modern-day GPUs, which means that the resulting images can be interactively manipulated and explored. It is indicated that how a combined use of registration, segmentation and 3D visualization can make the data more easily, accurately and intuitively explorable. The techniques are meant to aid in the understanding of human anatomy, pathology, and in the diagnosis of related medical conditions. Classification using a multidimensional transfer function is as shown in fig. 5. The segmented tumor from one MRI data set is visible among other soft tissues rendered from a different MRI data set.

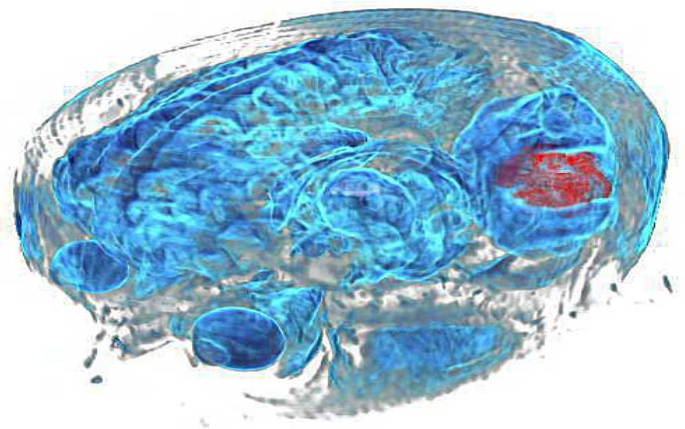

Fig 5 Classification using a multidimensional transfer functions.

\section{MARCHING CUBES [8]}

The next method [8] studied by W. Narkbuakaew, S. Sotthivirat, D. Gansawat, P. Yampri, K. Koonsanit, W. Areeprayolkij, W. Sinthupinyo, and S. Watcharabutsarakham, is "3d Surface Reconstruction Of Large Medical Data Using Marching Cubes In Vtk". A three-dimensional surface is a representation of volumetric image data in a shape form. One algorithm, which is acceptable for reconstructing a three dimensional surface, is the Marching cubes. It uses patterned cubes or isosurface to approximate contours. The marching cubes algorithm needs some processes or algorithms to reduce time and memory for reconstructing a surface from large volumetric data.

A common way to solve this problem is by subsampling or reducing a volumetric image size, but the quality of the reconstructed three-dimensional surface will be poor if only apply subsampling is applied. Due to the effect of volumetric subsampling, a process to improve the quality of a surface reconstructed from the sampled volumetric data is proposed. It is based on a pipeline of Visualization Toolkit (VTK). Proposed approach includes three main steps, which are preprocessing, reconstructing, and displaying. In this paper there is focus on the preprocessing step including sampling, thresholding and Gaussian filtering. Specifically, the effect of the subsampling factors, and the standard deviation parameters for Gaussian filtering are studied. Furthermore, time and memory usages are discussed in this research as well. The experimental results show that the proposed process can be applied effectively to reconstruct a three dimensional surface from large volumetric image data. 
In this research, a process for reconstructing a threedimensional surface from large volumetric image data is proposed. The process is based on the pipeline of VTK. In the preprocessing step before reconstructing the surface, sub sampling is applied, thresholding, and Gaussian smoothing to reduce the computation time. The experimental results show that the Gaussian smoothing step is necessary to reduce some noises, which may occur in the previous thesholding step; however, too much Gaussian smoothing may reduce the quality of a three dimensional surface. The last experiment shows that the time and memory usages for reconstructing a surface by the proposed process are less than directly reconstructing the $3 \mathrm{D}$ surface without subsampling. The quality of 3D surface reconstruction by subsampling large volumetric data is satisfactory if the appropriate parameters of the subsampling factor and the standard deviation are chosen. Thus, the proposed process can effectively reconstruct a threedimensional surface from large volumetric image data with limited resources.

\section{USE OF ISSSVM [9]}

Next [9] method by Lei Guo, Ying Li, Dongbo Miao, Lei Zhao, Weili Yan, and XueqinShen is , "3-D Reconstruction of Encephalic Tissue in MR Images Using Immune Sphere shaped SVMs." This method is studied to be more effective. It is mentioned that the Support vector machines (SVMs) are effective methods for the general purpose of pattern recognition. Initially, SVM was proposed to be a plane-shaped two-class classifier. With the development of the SVM theory, the sphere-shaped SVMs (SSSVMs) were proposed for solving the classification problems of the class-imbalance. It is mentioned that the SSSVM is extended its application to the 3 -D reconstruction. The main idea is that the SSSVM can transform an original 3-D object space into a high dimensional feature space by the kernel function and construct a compact hyper-sphere which encloses the target object in the feature space. The data which are on or near the surface of the hypersphere are support vectors (SVs). Thus, the model of a 3-D object and data which support the surface in the original space are obtained. This approach has multiple advantages. First, through the flexible hyper-sphere in the feature space, a 3-D object model can be reconstructed no matter how arbitrarily irregular the object surface is in the original space. In addition, there is un-necessity for priori information regarding the shape or the topology of an object. Selecting parameters for the SSSVM and the kernel function, however, is a complicated issue. Appropriate parameters can make the model more flexible and can help to obtain more accurate data description. Immune algorithms (IA) have the abilities of memorizing and self-adaptively adjusting. In this study, IA is introduced in searching for the optimal parameters. Combining SSSVM with IA, Immune SSSVM (ISSSVM) is used in 3-D reconstruction of the encephalic tissue in MR images. In the experiment, the seven objects were reconstructed by the ISSSVM respectively. Segmentation results of 112th slice are as shown in figure 6 . In the brain MR images, the highly irregular surface of each encephalic tissue is a real challenge to the traditional 3-D reconstruction algorithms. Due to its advantage in solving nonlinear problems, SSSVM was applied in the 3-D image reconstruction. In order to reduce the blindness of the parameter selection and to perfect the SVM theory, IA was introduced in searching for the optimal parameters.

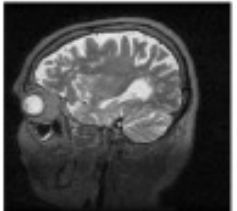

(a)

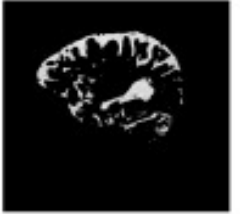

(b)

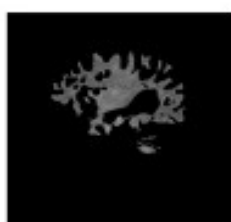

(c)
Fig.6. Segmentation results of 112th slice. (a) Original slice. (b) Cerebral spinal fluid. (c) Cerebral white matter.

The Visual effects of 3-D reconstruction which refers to background, scalp, osseous compact substance, osseous spongy substance, cerebral spinal fluid, cerebral gray matter and cerebral white matter are shown in figure 7.
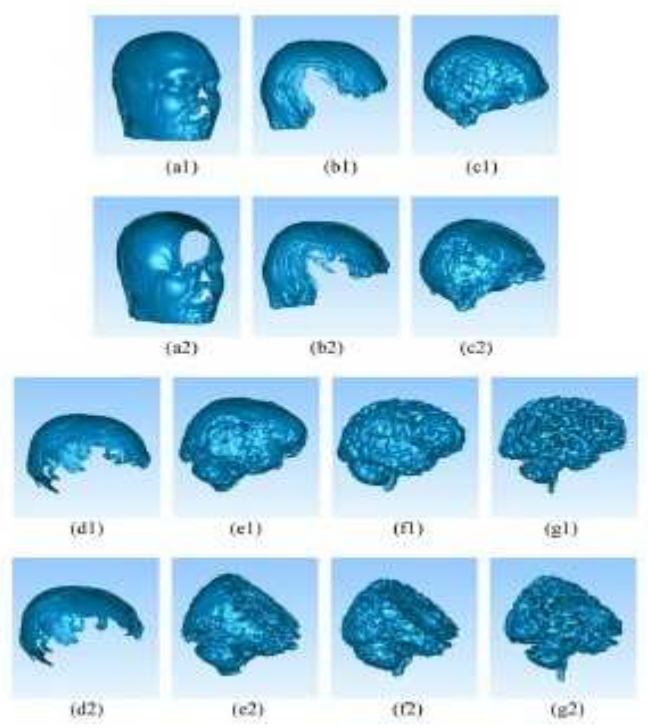

Fig.7 Visual effects of 3-D reconstruction (a) refers to background;(b) refers to scalp; (c) refers to osseous compact substance; (d) refers to osseous spongy substance; (e2) refers to cerebral spinal fluid; (f2) refers to cerebral gray matter; (g) refers to cerebral white matter. (a1) ISSSVM, (b1)

ISSSVM,(c1) ISSSVM, (a2) LISSSVM, (b2) LISSSVM, (c2) LISSSVM, (d1) ISSSVM,(e1) ISSSVM, (f1) ISSSVM, (g1) ISSSVM, (d2) LISSSVM, (e2) LISSSVM,(f2) LISSSVM, (g2) LISSSVM. 
In this study, ISSSVM was investigated and the proposed approach could reconstruct complicated objects efficiently without pre- or post-processing and supplementary geological information. Theoretical analysis and experimental results indicated that ISSSVM exhibits its great potential in 3-D image reconstruction, especially for cases in medical images with complicated tissue boundaries.

\section{CONCLUSIONS}

Different methods of converting 2D MRI brain images into 3D images are studied. The last method studied which is use of immune SSSVM is studied to be more appropriate as the results are more accurate and the time required is also less.

\section{REFERENCES}

[1]. K Narayanan 1, Yogesh Karunakar2, “3-D Reconstruction of Tumors in MRI Images", International Journal of Research and Reviews in Signal Acquisition and Processing IJRRWSAP,Vol. 1, No. 2, June 2011

[2]. Hassan Khotanloua , Olivier Colliotb , Jamal Atifc , Isabelle Blocha, "3D brain tumor segmentation in MRI using fuzzy classification, symmetry analysis and spatially constrained deformable models", Science Direct,Fuzzy Sets and Systems 160 (2009) 1457 - 1473

[3]. Cheng-Sheng Hung, Chien-Feng Huang, Ming Ouhyoung, "Fast Volume Rendering for Medical Image Data", Communication and Multimedia Laboratory Department of Computer Science and Information Engineering National Taiwan University, Taiwan.

[4]. Duška Kleut, Milorad Jovanović and prof. Dr Branimir Reljin, “3D Visualisation of MRI images using MATLAB", Journal Of Automatic Control, University Of Belgrade, VOL. 16:1-3, 2006

[5]. Lei Wang, Guizhi Xu, Lei Guo, Xuena Liu and Shuo Yang, "3D Reconstruction of Head MRI Based on One Class Support Vector Machine with Immune algorithm", Proceedings of the 29th Annual International Conference of the IEEE EMBS Cité International, Lyon, France August 2326, 2007.

[6]. Jayram Udupa, Hsiu-Mei Hung, Keh-Shih Chuang, "Surface and Volume Rendering in Three- Dimensional Imaging"

[7]. Paul Herghelegiu, Marius Gavrilescu, Vasile Manta, "Visualization of Segmented Structures in 3D Multimodal Medical Data Sets", Advances in Electrical and Computer Engineering, Volume 11, No. 3, 2011

[8]. W. Narkbuakaew, S. Sotthivirat, D. Gansawat, P. Yampri, K. Koonsanit, W. Areeprayolkij, W. Sinthupinyo, and S. Watcharabutsarakham, "3d Surface Reconstruction Of Large Medical Data Using Marching Cubes In Vtk" National

Electronics and Computer Technology Center, Phahon Yothin Rd, Klong Luang, Pathumthani, Thailand

[9]. Lei Guo, Ying Li, Dongbo Miao, Lei Zhao, Weili Yan, and XueqinShen, "3-D Reconstruction of Encephalic Tissue in MR Images Using Immune Sphere shaped SVMs", IEEE Transactions on Magnetics, Vol.47, No.5, May 2011.

\section{BIOGRAPHIES}

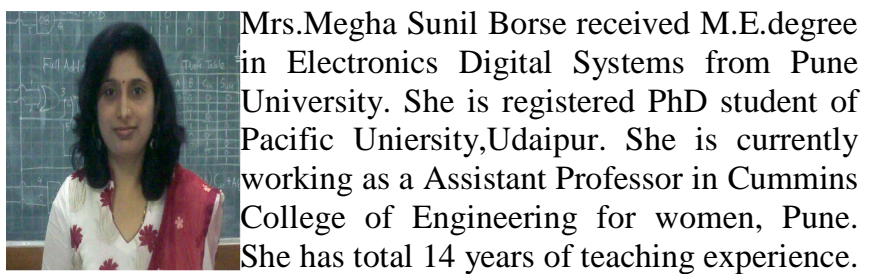
Her area of specialization is biomedical image processing.

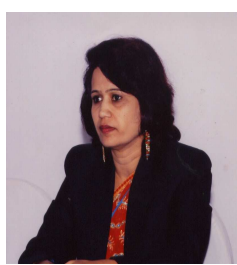

Dr. Shubhangi B. Patil had completed her $\mathrm{PhD}$ degree in Electronics Engineering. She is working as a professor and P G coordinator in JJ Magdum College of Engineering,Sangli. She has total 18 years of teaching experience and also has membership of professional societies like LM ISTE,IETE, Fellow member of "International society of Biotechnology"

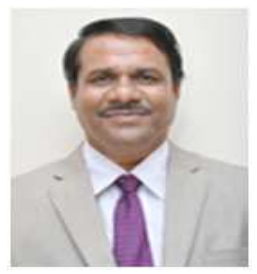

Prof. B.S. Patil received Msc degree in Electronics also he is pursuing his M.E. degree. He is currently working as Associate Professor and Head of Department of Information and Technology Department in PVPIT, Budhgaon. $\mathrm{He}$ has total teaching experience of 23 years. His area of specialization is Television and Communication Engineering. 